\title{
Comparing Augmentation with Non-Antidepressants over Sticking to Antidepressants after Treatment Failure in Depression: A Naturalistic Study
}

Authors

Affiliations
S. Köhler ${ }^{1, *}$, T. Unger ${ }^{2, *}$, S. Hoffmann ${ }^{2, *}$, B. Steinacher ${ }^{3, *}$, T. Fydrich ${ }^{4, *}$, T. Bschor B $^{5}$ *

Affiliation addresses are listed at the end of the article
Key words

depression

depression treatment

antidepressants $\begin{array}{ll}\text { received } & 24.12 .2011 \\ \text { revised } & 16.07 .2012 \\ \text { accepted } & 24.07 .2012\end{array}$

Bibliography

Dol http://dx.doi.org/

10.1055/s-0032-1323677

Published online ahead of print:

October 23, 2012

Pharmacopsychiatry 2013;

46: 69-76

(c) Georg Thieme Verlag KG

Stuttgart · New York

ISSN 0176-3679

\section{Correspondence}

\section{S. Köhler, MD}

Department of Psychiatry and Psychotherapy

Charité University Medicine

Berlin

Campus Mitte

Charitéplatz 1

10117 Berlin

Germany

Tel.: +49/30/450 617195

Fax: $+49 / 30 / 450517944$

stephan.koehler@charite.de

\section{Abstract}

$\nabla$

Introduction: Non-response to an antidepressant monotherapy in unipolar depression is quite common. Therefore strategies for subsequent treatment steps are necessary. However, there is a lack of direct comparisons of these different strategies. In this naturalistic study we compared the outcome to different strategies after failure of the primary antidepressant treatment.

Methods: Failure of primary antidepressant monotherapy occurred in 135 patients. 98 of these patients have been administered 4 treatment strategies of the physicians' choice: lithium augmentation (Li-Augm), switching to another antidepressant (AD-Switch), combination of 2 antidepressants (AD-Comb) or augmentation with second generation antipsychotic (SGA-Augm). Primary outcome measure was the 17-item Hamilton rating scale for depression (HRSD).

\section{Introduction}

\section{$\nabla$}

A main result of the Sequenced Treatment Alternatives to Relieve Depression (STAR * D) trial [1] was that outpatients who initially received monotherapy with citalopram only showed remission in $28 \%$ of the cases. Nevertheless, remission should be the aim of treatment [2]. Consequently failure to primary antidepressant treatment is quite common. However, strategies for second treatment steps, especially in comparison to each other, have rarely been investigated [3].

There are 4 frequently used pharmacological strategies for treatment of depression after initial antidepressant monotherapy showed no response: Lithium augmentation, switch of antidepressants, combination of antidepressants and

All authors contributed to and have approved the final manuscript.
Results: Patients who received Li-Augm or augmentation with SGAs showed significantly greater improvement in HRSD and BDI compared to patients with antidepressant switch or antidepressant combination. Remission rates for LiAugm and SGA-Augm were $89.3 \%$ and $86.2 \%$ compared to $40.7 \%$ for AD-Switch and $42.9 \%$ for AD-Comb.

Discussion: Changing to another pharmacological class (Li-Augm or augmentation with SGAs) showed better treatment results than sticking to the class of antidepressants (AD-Switch and ADComb) after primary failure in response to antidepressant monotherapy in unipolar depression. The lack of randomization and absence of a nonresponse definition are design flaws. Controlled studies are required to confirm the findings of this trial.

augmentation with second generation antipsychotics (SGA). Evidence for the effectiveness of these strategies varies and there are hardly any data analyzing superiority of one treatment step over the other [4]. Especially empirically evidence for antidepressant switch or antidepressant combination is poor [5-8]. In contrast, there are more studies showing a better level of evidence for augmentation strategies with lithium or SGA: Efficacy of lithium-augmentation was shown in a meta-analysis of 10 studies [9]. Odds ratio (OR) for probability of response to lithium was 3.11 compared to placebo and a number needed to treat (NNT) of 5. Also SGA was found to be a reasonable augmentation strategy for nonresponse to antidepressant (AD) monotherapy. In a meta-analysis including 16 studies with 3480 patients, Nelson and Papakostas (2009) found that augmentation with 4 SGA (aripiprazole, olanzapine, risperidone, quetiapine) was supe- 


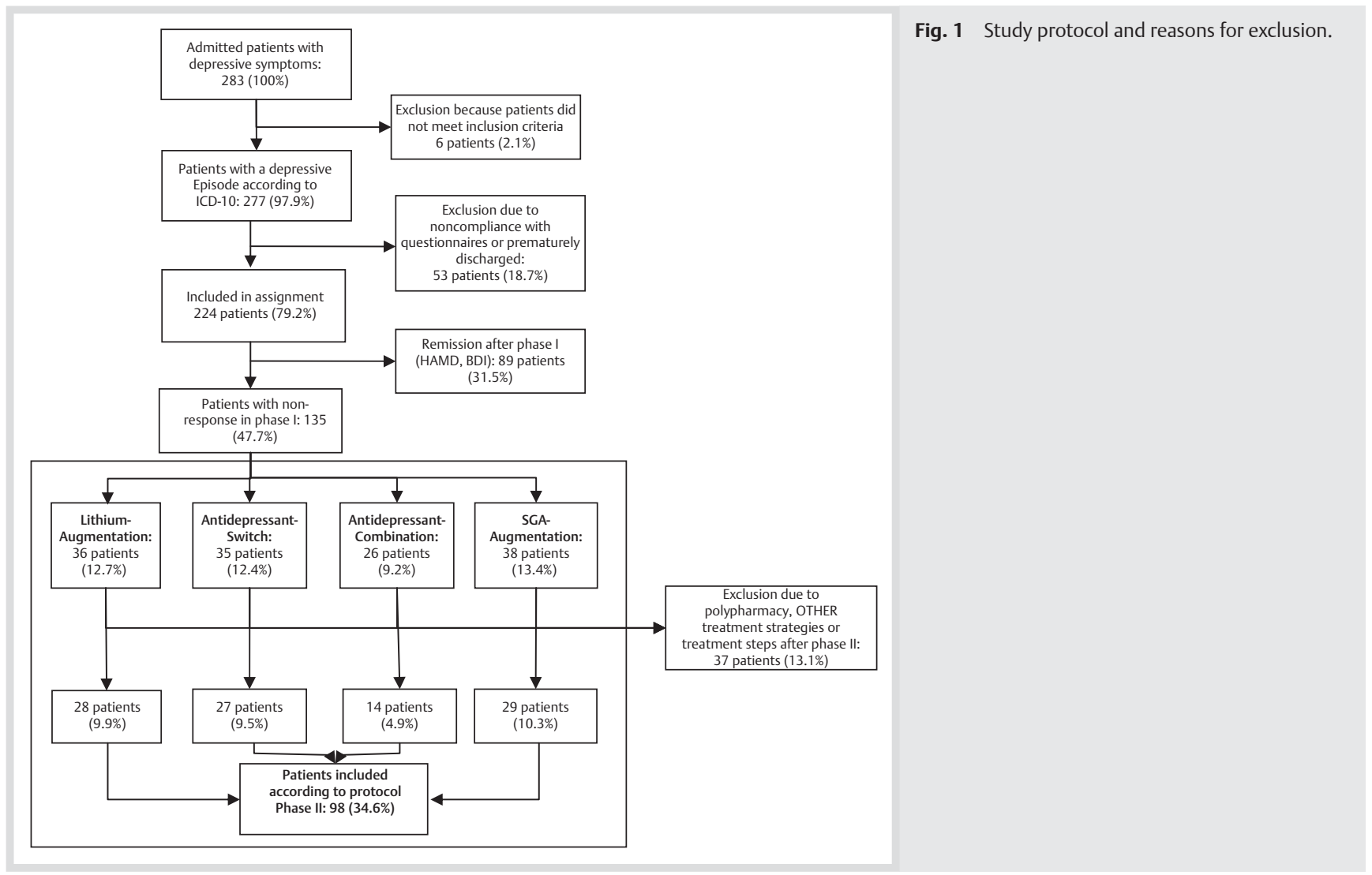

rior to augmentation with placebo [10]. The OR for response to SGA vs. placebo was 1.69 and an NNT of 9 patients was calculated.

In conclusion, the evidence for differences regarding the efficacy of different pharmacological treatment strategies after failure to $\mathrm{AD}$ monotherapy varies and there are hardly any competitive investigations of these strategies [11]. Therefore further insight not only from RCTs but also from naturalistic trials of everyday clinical routine are warranted [12]. Particularly results gained from trials in clinical practice are required because the patient population of randomized controlled trials (RCTs) is often found to be biased $[13,14]$. However RCTs are the basis of advancing knowledge. Due to the lack of RCTs on this issue, the aim of this study was to find differences in treatment response after failure of primary antidepressant monotherapy in a naturalistic design (lithium-augmentation, antidepressant-combination, switch of antidepressants, augmentation with SGA).

\section{Methods \\ $\nabla$}

The study was naturalistic in design and treatment was implemented according to the special needs of each patient. Treatment was not influenced due to the design of the study and analyses have been performed post-hoc. Over a period of 2 years all patients with a unipolar depression were included in the trial. The entry criteria included: (i) ICD-10 criteria for a major depressive episode or recurrent depression as the principal current diagnosis, (ii) aged 18 or older, (iii) informed consent from the patient, (iv) inpatient treatment duration $\geq 21$ days [15]. After admission, the diagnosis of a depressive episode was done by the attending physicians within 3 days according to ICD-10 using the International Diagnostic Checklist [16]. Participants were excluded (i) if they had current severe alcohol or drug dependence (participants with mild to moderate alcohol or drug dependence were included), (ii) if they had a previous history of schizophrenia, schizoaffective disorder, bipolar I disorder (a history of hypomania was permitted), (iii) if the depressive episode was attributable to organic illness and (iv) if they had such severe concentration deficits to complete the questionnaires at admission (e.g.), had language difficulties or did not complete the questionnaires at discharge ( $\bullet$ Fig. 1). The study has been conducted in accordance with the current version of the Declaration of Helsinki and was approved by the local Ethics Committee. Patients were treated under consideration of the German S3 guideline for treatment of depression [17]. All psychiatric drugs, the medication class, the dosage and the treatment duration were recorded systematically. In addition, the duration and type of other treatments like psychotherapy, occupational therapy, physical therapy or art therapy were recorded.

\section{Assignment}

Only patients with an initial antidepressant monotherapy with a duration of 21-35 days (phase I) were analyzed followed by an evaluation of the response to treatment by the attending clinicians. In 135 cases, patients showed an indication for change of treatment according to the clinical judgement (no defined measurement). 98 patients met criteria for assignment to one of the 4 treatment groups (phase II): 1) Lithium-augmentation (LiAugm), 2) switch of antidepressants (AD-Switch), 3) combination of antidepressants (AD-Comb), 4) augmentation with SGA (SGA-Augm). Criteria for enrolment into analysis were: patients had to have an adequate antidepressant monotherapeutic treatment in phase I according to duration (21-35 days) and dosage ( $\bullet$ Table 1, recommendations for doses of antidepressants, doses used in this study). Only experienced clinicians assigned the 
Table 1 Antidepressants and dose recommendation.

\begin{tabular}{|c|c|c|}
\hline & $\begin{array}{l}\text { Dose recommendation } \\
\text { according to guideline in } \mathrm{mg}\end{array}$ & $\begin{array}{l}\text { Doses used in } \\
\text { this study in } \mathrm{mg}\end{array}$ \\
\hline venlafaxine & $75-225$ & $150-300$ \\
\hline duloxetine & 60 & $60-120$ \\
\hline mirtazapine & $15-45$ & $30-45$ \\
\hline paroxetine & $20-40$ & $20-40$ \\
\hline escitalopram & $10-20$ & $10-20$ \\
\hline fluoxetine & $20-40$ & $40-60$ \\
\hline clomipramin & $100-250$ & $150-225$ \\
\hline amitriptyline & $100-300$ & $150-250$ \\
\hline doxepin & $100-300$ & $100-200$ \\
\hline sertraline & $50-100$ & $100-150$ \\
\hline
\end{tabular}

patients to the appropriate treatment group according to the patients' medical/psychiatric needs. Change of medication was not caused by side effects of the phase I treatment. There were no other psychotropic drugs in phases I and II other than the medication of the treatment groups. Treatment in phase II was adequate in doses and duration. Patients who received a third treatment step due to failure in phase II were not included in the analysis. To assess the severity of depressive symptoms, clinician-rated and self-rating instruments were used at admission and at discharge ( $\mathrm{t} 1$ and $\mathrm{t} 2$ ). Treatment groups were as heterogeneous as they normally appear in clinical practice.

\section{Diagnostic methods}

The severity of depressive symptoms was measured using clinician-rated and self-rated assessment procedure. The Hamilton rating scale for depression $\left(\mathrm{HRSD}_{17}\right)$ was used to assess the severity of depressive symptoms [15] and was the primary outcome criterion. It was performed by trained psychologists who were blinded regarding the treatment arm. Secondary measures included the Beck depression inventory [18] and the Brief symptom inventory (BSI) to assess the patients' depressive and general psychological symptoms as means of self-assessment [19]. The attending physicians rated the patient's global level of functioning using the Global assessment of functioning scale (GAF) [20] as well as the global severity of the psychiatric disorder using the Clinical global impression scale (CGI) [21]. The assessment of personality disorders was performed using the structured clinical interview for DSM-IV personality disorders (SKID II) and was conducted by trained and experienced clinical psychologists 3 weeks after admission [22]. All raters were given training and supervision to perform clinician-rated assessment procedures (HRSD, CGI, GAF, SKID II).

\section{Statistics}

To analyze if patients in the 4 treatment groups were comparable, analysis of variance followed by post-hoc tests (parametric data) or Kruskall-Wallis test followed by Mann-Whitney U-test (non-parametric data) for important psychometric characteristics were performed. Significant differences were added as covariates in the following analyses. The primary analysis for examining changes over time was a $2 \times 2$ ANCOVA with the 2 factors "treatment" and "time", controlling for pre-treatment scores (repeated-measure ANCOVA). Afterwards, post-hoc analyses for differences between treatment groups were performed. All significance tests were performed at a 2-tailed alpha level of 0.05, incorporating an LSD procedure for multiple comparisons.
For all tests, effect sizes (ES) for independent random samples were calculated using Cohen's d statistic and the effect sizes for repeated measures [23]. At the end of treatment, the remission rates under the 4 treatment conditions were compared using 2-sided Pearson's $X^{2}$ tests (remission: HRSD <8) [24]. The statistical analyses were carried out using SPSS 17.

\section{Results}

\section{$\nabla$}

A total of 283 patients were screened. 277 patients met the inclusion criteria and 224 patients were enrolled in the study. 135 patients (47.7\%) showed no response after initial antidepressant monotherapy and were therefore assigned to one of the 4 treatment groups. Another 37 patients (13.1\%) were excluded due to polypharmacy, different treatment strategies or treatment steps after phase II. Therefore 98 patients (34.6\%) were included in the study protocol. $\triangle$ Fig. 1 shows the study chart including reasons for drop out. Table 2 displays baseline demographic and illness characteristics for the entire sample. A severe depressive episode was the principal diagnosis in $43.7 \%$ of patients and a recurring severe depressive episode was diagnosed in $27.6 \%$ of all cases using ICD-10. There were no significant treatment group differences in baseline demographic or illness characteristics and comorbidities. The average HRSD score on admission was $28.2(S D=11.1)$ characterising a patient group with rather severe depressive symptoms. Antidepressant medication in phase I was similar in the treatment groups ( $\bullet$ Table 2). Change of treatment was performed after an average of 27.3 days (SD: 5.7) without differences between the treatment groups $(\mathrm{F}=1.27 ; \mathrm{p}=0.28)$. Characteristics of treatment in phase II including medication classes are displayed in $\bullet$ Table 3 and were also checked for duration and dosage. Again there was no difference between the treatment groups in terms of duration in phase II $(\mathrm{F}=1.02, \mathrm{p}=0.47)$. Additionally, patients took part in occupational therapy $(92 \%, n=206)$, additional cognitive-behavioural therapy ( $55.8 \%, n=125)$, cognitive-behavioural group therapy for anxiety disorders $(17 \%, n=38)$, music therapy $(25.4 \%$, $n=57)$, progressive muscle relaxation training $(17 \%, n=38)$, addiction therapy $(8 \%, n=18)$, physical and sports therapy $(81.7 \%, n=183)$ and dance- and motion therapy $(40.2 \%, n=90)$.

\section{Treatment effects}

Analysis of variance for repeated measures revealed a significant improvement and large effect sizes for all patients from baseline to discharge in all inventories (HRSD: $\mathrm{F}=648, \mathrm{p}=<0.001$; $\mathrm{BDI}$ : $\mathrm{F}=194.7, \mathrm{p}=<0.001$; BSI-GSI, $\mathrm{F}=167.59, \mathrm{p}=<0.0$ ). According to [23] effect sizes $d$ for repeated measurements indicate a substantial reduction $(d>0.80)$ of depressive symptoms $\left(E_{\text {HRSD }} d=3.2\right.$, $\mathrm{ES}_{\mathrm{BDI}}=1.9$ ) and general psychological distress $\left(\mathrm{ES}_{\mathrm{BSI}-\mathrm{GSI}}=1.6\right)$ during treatment. The interaction effect "time by treatment condition" indicated a significant difference in the amount of reduction of the depression severity measured using the HRSD between the 4 treatment groups (HRSD: $F=2.84, p=0.04$; BDI: $\mathrm{F}=3.68, \mathrm{p}=0.01$; $\triangle$ Table 4). The subsequent post-hoc analyses ( $\bullet$ Table 5) revealed a significantly higher improvement for $\mathrm{Li}-$ Augm over AD-Switch (mean difference [MD]: 2.72, $\mathrm{p}=0.02$ ) and for Li-Augm over $A D$-Comb $(M D=3.45, p=0.02)$ in the HRSD. Likewise SGA-Augm showed a significant higher improvement compared to $\mathrm{AD}-$ Switch $(\mathrm{MD}=2.34, \mathrm{p}=0.04)$ and $\mathrm{AD}$-Comb. $(\mathrm{MD}=3.07, \mathrm{p}=0.03)$. The BDI score showed similar results: Patients in the Li-Augm rated a higher improvement of their 
Table 2 Baseline demographic and sample characteristics of the patients.

\begin{tabular}{|c|c|c|c|c|c|c|c|c|}
\hline & & Li-Augm & \multicolumn{2}{|c|}{ AD-Switch } & AD-Comb & SGA-Augm & $F / x^{2}$ & $\mathbf{p}$ \\
\hline \multicolumn{2}{|l|}{ number of patients } & 28 & \multicolumn{2}{|c|}{27} & 14 & 29 & & \\
\hline \multicolumn{2}{|l|}{ age in y (SD) } & $50.76(13.73)$ & \multicolumn{2}{|c|}{$57.97(11.06)$} & $50.47(12.41)$ & $50.89(11.95)$ & $F=3.0$ & 0.75 \\
\hline \multicolumn{2}{|l|}{ range } & $26-64$ & \multicolumn{2}{|c|}{$26-65$} & $30-68$ & $33-76$ & & \\
\hline \multicolumn{2}{|l|}{ female n (\%) } & $17(60.71)$ & \multicolumn{2}{|c|}{$16(59.26)$} & $9(64.3)$ & $18(62.07)$ & $x^{2}=0.06$ & 0.997 \\
\hline \multirow[t]{3}{*}{ education n (\%): } & apprenticeship & $13(46.4)$ & \multicolumn{2}{|c|}{$11(40.7)$} & $9(64.3)$ & $14(48.3)$ & & \\
\hline & graduate degree & $15(53.5)$ & \multicolumn{2}{|c|}{$16(59.2)$} & $4(28.5)$ & $14(48.2)$ & $x^{2}=0.59$ & 0.89 \\
\hline & other & $1(3.6)$ & \multicolumn{2}{|l|}{0} & $1(7.1)$ & $1(3.6)$ & & \\
\hline \multirow[t]{3}{*}{ working situation n (\%) } & working & $12(42.8)$ & \multicolumn{2}{|c|}{$8(29.6)$} & $4(28.5)$ & $9(31.3)$ & $x^{2}=1.42$ & 0.7 \\
\hline & unemployed & $6(21.4)$ & \multicolumn{2}{|c|}{$1(3.7)$} & $5(35.7)$ & $9(31.3)$ & & \\
\hline & other & $10(36.4)$ & \multicolumn{2}{|c|}{$18(66.7)$} & $5(35.7)$ & $11(37.9)$ & & \\
\hline \multirow[t]{5}{*}{ family status, $\mathrm{n}(\%)$} & single & $3(10.7)$ & \multicolumn{2}{|c|}{$2(7.4)$} & $5(35.7)$ & $9(31.0)$ & & \\
\hline & married & $15(53.5)$ & \multicolumn{2}{|c|}{$15(55.6)$} & $1(7.1)$ & $13(44.8)$ & $x^{2}=10.0$ & 0.02 \\
\hline & separated & $5(17.9)$ & \multicolumn{2}{|c|}{$3(28.6)$} & $4(28.5)$ & $1(3.6)$ & & \\
\hline & divorced & $4(14.3)$ & \multicolumn{2}{|c|}{$5(18.5)$} & $3(21.4)$ & $5(18.5)$ & & \\
\hline & widowed & $1(3.6)$ & \multicolumn{2}{|c|}{$2(7.4)$} & $1(7.1)$ & $1(3.6)$ & & \\
\hline \multicolumn{2}{|l|}{ comorbidity axis I, n (\%) } & $10(36.4)$ & \multicolumn{2}{|c|}{$5(18.5)$} & $6(42.8)$ & $12(41.9)$ & $x^{2}=8.15$ & 0.06 \\
\hline \multicolumn{2}{|l|}{ comorbidity axis II } & $11(39.3)$ & \multicolumn{2}{|c|}{$9(28.0)$} & $4(28.6)$ & $11(37.9)$ & $x^{2}=6.57$ & 0.08 \\
\hline \multicolumn{2}{|l|}{$\begin{array}{l}\text { duration of treatment, in } \\
d(S D)\end{array}$} & $56.89(16.85)$ & \multicolumn{2}{|c|}{$50.74(17.12)$} & $54.36(14.88)$ & $57.55(17.37)$ & $F=3.0$ & 0.09 \\
\hline \multicolumn{2}{|l|}{$\begin{array}{l}\text { duration of treatment in } \\
\text { phase I in } d(S D)\end{array}$} & $26.11(2.07)$ & \multicolumn{2}{|c|}{28.89 (3.09) } & $27.93(2.52)$ & $26.11(3.07)$ & $F=1.27$ & 0.28 \\
\hline treatment of phase I & SSRI & $4(14.3)$ & & & $8(27.6)$ & $8(27.6)$ & $x^{2}=0.56$ & 0.91 \\
\hline & SSNRI & $5(17.9)$ & & $0.7)$ & $9(64.3)$ & $11(27.6)$ & $x^{2}=0.04$ & 1.0 \\
\hline & TCA & $3(10.7)$ & & & $4(28.5$ & $6(33.3$ & $x^{2}=0.45$ & 0.36 \\
\hline & mirtazapine & $2(7.4)$ & & & $6(40.0)$ & $3(15.3)$ & $x^{2}=0.12$ & 0.99 \\
\hline diagnosis & moderate depress & isode (F 32.1) & $0(0)$ & $3(28.6)$ & $1(7.1)$ & $3(15.3)$ & & \\
\hline & severe depressive & de (F 32.2) & $16(57.1)$ & $15(55.6)$ & $7(49.9)$ & $13(44.8)$ & & \\
\hline & $\begin{array}{l}\text { recurrent depress } \\
\text { episode moderat }\end{array}$ & $\begin{array}{l}\text { sorder, current } \\
\text {.1) }\end{array}$ & $0(0)$ & $0(0)$ & $0(0)$ & $0(0)$ & & \\
\hline & $\begin{array}{l}\text { recurrent depress } \\
\text { episode severe (F }\end{array}$ & sorder, current & $12(42.9)$ & $9(28.0)$ & $6(42.8)$ & $10(35.0)$ & & \\
\hline
\end{tabular}

depressive symptoms compared to AD-Switch $(\mathrm{MD}=3.32$, $\mathrm{p}=0.04)$ and $\mathrm{AD}$-Comb. $(\mathrm{MD}=6.32, \mathrm{p}<0.01)$. Patients augmented with SGA also showed a greater reduction in the BDI than patients with $A D-C o m b(M D=4.03, p=0.04)$, but not compared to AD-Switch $(\mathrm{MD}=1.03, \mathrm{p}=0.56)$. There were no significant differences between Li-Augm and SGA-Augm in the HRSD or the BDI. Regarding the amount of reduction in general psychological symptoms (BSI-GSI) significant differences between the treatment groups could be found (BSI-GSI, F=2.87; p=0.04). Patients with Li-Augm and SGAAugm had higher amount of reduction than patients with $\mathrm{AD}-\mathrm{Comb}$ (Li-Augm: $\mathrm{MD}=0.44, \mathrm{p}<0.01$, SGA-Augm: $\mathrm{MD}=0.31, \mathrm{p}=0.03$ ).

\section{Remission rates}

Comparing the remission rates between the 4 treatment conditions, patients with Li-Augm showed higher remission rates $(89.3 \%)$ in the HRSD with a statistical trend $(\mathrm{p}<0.1)$ compared to AD-Switch $\left(40.7 \% ; X^{2}=3.07 ; p=0.08\right)$ but not to AD-Comb (ADComb: $\left.42.9 \% ; X^{2}=1.75, p=0.19\right)$. The SGA-Augm-group also had higher remission rates $(86.2 \%)$ compared to $A D$-Switch $\left(X^{2}=2.82\right.$,

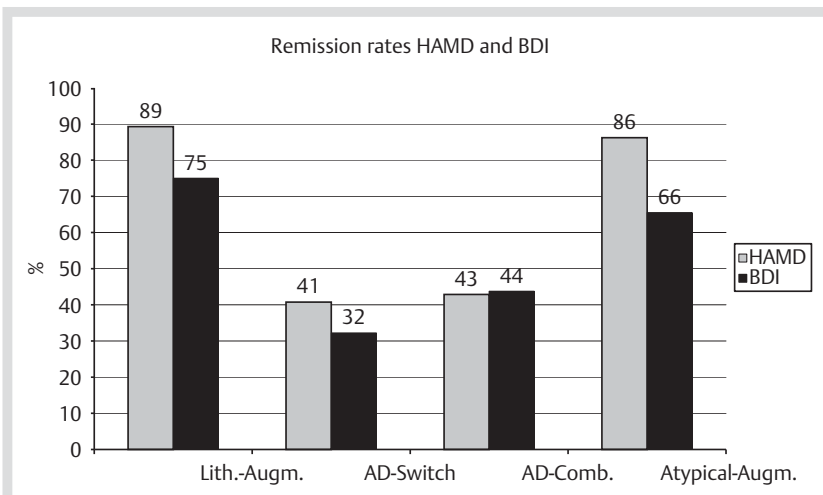

Fig. 2 Remission rates in HAMD and BDI in \% comparing phase II patients only. 
$\mathrm{p}=0.09)$ but not to AD-Comb $\left(\mathrm{X}^{2}=1.59, \mathrm{p}=0.21\right)$. All remission rates are displayed in $\bullet$ Fig. 2.

\section{Global functioning and clinical global impression}

Overall functioning improved considerably from baseline to discharge (GAF: $\mathrm{F}=250.1, \mathrm{p}<0.001, \mathrm{ES}_{\text {pre-post }}: \mathrm{d}=2.3$ ) for all patients. Also there was a significant reduction in general severity of disorder from intake to discharge (CGI: $\mathrm{F}=524.2, \mathrm{p}<0.001$, $\mathrm{ES}_{\text {pre-post }}: \mathrm{d}=3.4$ ). The interaction effect "time by treatment con- dition" indicated significant differences between treatment conditions regarding the amount of reduction from baseline to discharge (GAF: $F=5.21, p=0.002$; CGI: $F=4.28, p=0.007$; $\odot$ Table 3). Posthoc analyses ( $\odot$ Table 4 ) again revealed a higher gain of functioning (GAF) for the Li-Augm compared to AD-Comb (MD=0.78, $\mathrm{p}<0.01$ ). Patients in the SGA-Augm group also had a higher improvement compared to AD-Switch and AD-Comb (AD-Comb: $\mathrm{MD}=0.90$, $\mathrm{p}<0.01$ [trend]; AD-Switch: $\mathrm{MD}=0.37, \mathrm{p}=0.08$ ). Improvement in GAF did not differ between Li-Augm and SGA-Augm. Both groups

Table 3 Treatment in phase II.

\begin{tabular}{|c|c|c|c|c|c|c|c|}
\hline & Li-Augm & AD-Switch & & AD-Comb & & \multicolumn{2}{|c|}{ Atypical-Augm. } \\
\hline number of patients & 27 & 27 & \multicolumn{3}{|c|}{14} & \multicolumn{2}{|c|}{28} \\
\hline specific characteristics & average lithium level: & switch-strategy: & $n$ & combination strategy: & $\mathrm{n}$ & \multicolumn{2}{|c|}{ SGA: } \\
\hline \multirow[t]{9}{*}{ of strategy: } & $0.82 \mathrm{mmol} / \mathrm{L}$ & SSRI $\rightarrow$ SSNRI: & $5(18.5 \%)$ & SSRI-SSNRI & $3(21.4 \%)$ & amisulpride & $3(10.3 \%)$ \\
\hline & & SSRI $\rightarrow$ mirtazapine & $2(7.4 \%)$ & SSRI-mirtazapine & $1(7.1 \%)$ & aripiprazole & $5(17.2 \%)$ \\
\hline & & SSNRI $\rightarrow$ mirtazapine & $4(14.8 \%)$ & SSRI-TCA & $1(7.1 \%)$ & clozapine & $2(6.9 \%)$ \\
\hline & & SSNRI $\rightarrow$ TCA & $4(14.8 \%)$ & SSNRI-mirtazapine & $3(21.4 \%)$ & olanzapine & $7(24.1 \%)$ \\
\hline & & SSNR $\rightarrow$ SSRI & $2(7.4 \%)$ & SSNRI-SSRI & $2(14.2 \%)$ & quetiapine & $7(24.1 \%)$ \\
\hline & & TCA $\rightarrow$ SSNRI & $1(3.7 \%)$ & TCA-SSNRI & $2(14.2 \%)$ & risperidone & $3(10.3 \%)$ \\
\hline & & $\mathrm{TCA} \rightarrow \mathrm{SSRI}$ & $5(18.5 \%)$ & mirtazapine-SSRI & $2(14.2 \%)$ & ziprasidone & $2(6.9 \%)$ \\
\hline & & mirtazapine $\rightarrow$ SSNRI & $3(11.1 \%)$ & & & & \\
\hline & & mirtazapine $\rightarrow$ TCA & $1(3.7 \%)$ & & & & \\
\hline
\end{tabular}

Li-Augm, lithium augmentation; AD-Switch, antidepressant switch; AD-Comb, antidepressant combination, SGA-Augm, augmentation with second generation antipsychotics; SSRI, selective serotonin reuptake inhibitors; TCA, tricyclic antidepressants; SNRI, serotonin-norepinephrine reuptake inhibitors; AD, antidepressants; SD, standard deviation; n, numbers

Table 4 Baseline and post-treatment scores.

\begin{tabular}{|c|c|c|c|c|c|c|c|c|c|}
\hline & & & & & & & $\begin{array}{l}\text { Interaction facto } \\
\text { vs. time" }\end{array}$ & 5 "tr & tment \\
\hline & & Total & Li-Augm & AD-Switch & AD-Comb & SGA-Augm & $\begin{array}{l}\text { F (adj.Greenh.- } \\
\text { Geisser) }\end{array}$ & df & p \\
\hline \multirow[t]{2}{*}{ HAMD } & baseline mean (SD) & $28.22(6.83)$ & $27.68(8.33)$ & $29.04(6.62)$ & $29.5(6.47)$ & $27.38(5.67)$ & 2.84 & 1 & 0.04 \\
\hline & $\begin{array}{l}\text { post-treatment } \\
\text { mean (SD) }\end{array}$ & $7.52(4.61)$ & $5.36(3.86)$ & $9.44(4.48)$ & $10.43(5.54)$ & $6.41(3.56)$ & & & \\
\hline \multirow[t]{2}{*}{ BDI } & baseline mean (SD) & $28.58(11.05)$ & $27.25(11.08)$ & $26.78(9.02)$ & $31.29(9.96)$ & $30.24(13.11)$ & 3.68 & 1 & 0.01 \\
\hline & $\begin{array}{l}\text { post-treatment } \\
\text { mean (SD) }\end{array}$ & $11.77(6.31)$ & $8.11(5.18)$ & $15.22(4.91)$ & $16.71(6.41)$ & $9.69(5.4)$ & & & \\
\hline \multirow[t]{2}{*}{ BSI-GSI } & baseline mean (SD) & $1.44(0.63)$ & $1.33(0.52)$ & $1.34(0.45)$ & $1.8(0.5)$ & $1.48(0.85)$ & 2.87 & 1 & 0.04 \\
\hline & $\begin{array}{l}\text { post-treatment } \\
\text { mean (SD) }\end{array}$ & $0.64(0.41)$ & $0.49(0.34)$ & $0.71(0.4)$ & $0.91(0.52)$ & $0.6(0.38)$ & & & \\
\hline \multirow[t]{2}{*}{ CGI } & baseline mean (SD) & $5.51(0.58)$ & $5.41(0.57)$ & $5.56(0.64)$ & $5.64(0.5)$ & $5.48(0.57)$ & 4.28 & 1 & $<0.01$ \\
\hline & $\begin{array}{l}\text { post-treatment } \\
\text { mean (SD) }\end{array}$ & $3.06(0.89)$ & $2.89(0.75)$ & $3.32(0.9)$ & $3.85(0.8)$ & $2.66(0.77)$ & & & \\
\hline \multirow[t]{2}{*}{ GAF } & baseline mean (SD) & $47.6(10.1)$ & $46.2(13.6)$ & $50(8.8)$ & $43.3(6.5)$ & $48.3(8.5)$ & 5.21 & 1 & $<0.01$ \\
\hline & $\begin{array}{l}\text { post-treatment } \\
\text { mean (SD) }\end{array}$ & $69.6(10.2)$ & $73.3(9.2)$ & $64.8(9.2)$ & $62.3(10.1)$ & $73.4(8.6)$ & & & \\
\hline
\end{tabular}

Li-Augm, lithium augmentation; AD-Switch, antidepressant switch; AD-Comb, antidepressant combination, SGA-Augm, augmentation with second generation antipsychotics; HAMD, Hamilton Depression; BDI, Beck Depression Inventory; BSI-CSI, Brief Symptom Inventory-Global Severity Index; CGI, Clinical Global Impression Scale, GAF, Global Assessment of Functioning; F values referring to the interaction of the factors "treatment" and "time" in the $2 \times 2$ ANCOVA, adjusted via Greenhouse-Geisser, df, degrees of freedom

\begin{tabular}{|c|c|c|c|c|c|}
\hline Post-Hoc-Tests & HAMD; $p$ & BDI, p & BSI-GSI, p & CGI, p & GAF, $p$ \\
\hline Li-Augm vs. AD-Switch & $0.02^{*}$ & $0.04^{*}$ & 0.32 & 0.25 & 0.25 \\
\hline Li-Augm vs. AD-Comb & $0.02^{*}$ & $0.03^{*}$ & $<0.01^{*}$ & $<0.01^{*}$ & $<0.01^{*}$ \\
\hline Li-Augm vs. SGA-Augm & 0.74 & 0.20 & 0.25 & 0.57 & 0.54 \\
\hline AD-Switch vs. AD-Comb & 0.61 & 0.18 & 0.32 & $0.02^{*}$ & $0.05^{*}$ \\
\hline SGA-Augm vs. AD-Switch & $0.04^{*}$ & 0.56 & 0.89 & 0.08 & 0.08 \\
\hline SGA-Augm vs. AD-Comb & $0.03^{*}$ & $0.04^{*}$ & $0.03^{*}$ & $<0.01^{*}$ & $<0.01^{*}$ \\
\hline \multicolumn{6}{|c|}{$\begin{array}{l}\text { ANCOVA, analysis of covariance; Li-Augm, lithium-augmentation; AD-Switch, antidepressant-switch; AD-Comb, antidepressant- } \\
\text { combination, SGA-Augm, augmentation with second generation antipsychotics; HAMD, Hamilton depression; BDI, Beck depression } \\
\text { inventory; BSI-GSI, brief symptom inventory-global severity index; CGI, clinical global impression scale, GAF, global assessment of } \\
\text { functioning, * , significance on the } 0.05 \text { level }\end{array}$} \\
\hline
\end{tabular}

Table 5 Post-hoc-tests for $2 \times 2$ ANCOVA. 
Li-Augm and SGA-Augm had significantly higher improvement in the CGI compared to AD-Comb (Li-Augm: $\mathrm{MD}=0.58, \mathrm{p}<0.01$; SGAAugm: $\mathrm{MD}=0.66, \mathrm{p}<0.01$ ) and to AD-Switch (Li-Augm: $\mathrm{MD}=0.29$, $\mathrm{p}=0.05$; SGA-Augm: $\mathrm{MD}=0.37, \mathrm{p}=0.01$ ).

\section{Safety measurements and compliance}

Adverse events reported by the patients were recorded. Somatic complaints were assessed and recorded.

There was no relevant difference between the groups in the percentage of patients in the safety set who reported at least one emergent adverse event on treatment $(51 \%)$. There were no deaths during the study and no suicide attempts. The most frequently affected systemic organ classes were gastrointestinal disorders (18.4\%) and nervous system disorders (20.5\%). The most frequent emergent adverse events were headache, nausea, and somnolence.

\section{Discussion}

$\nabla$

The present study investigated differences in treatment outcome between 4 different pharmacological treatment strategies after failure of the primary antidepressant monotherapy. In this naturalistic design, $60 \%$ of the depressive patients failed to show remission after primary treatment. This number is lower compared to the the STAR ${ }^{*} D$ trial, where $72 \%$ of patients failed to reach remission after citalopram monotherapy in treatment step one (1). Contrary to the STAR*D study, which only included outpatients, the present study only included inpatients. Nevertheless, overall results are comparable to those of other "effectiveness" studies investigating the quality of inpatient treatment of depression. In a multicenter trial of the German Research Network on Depression investigating suicidality in 1014 patients, response rates of $68.9 \%$ and remission rates of $51.9 \%$ in the HRSD could be achieved [25].

The second treatment step showed different response and remission rates depending on the treatment group. Generally, all patients showed relatively high response rates after treatment in step 2 (remission rate in HRDS: $30 \%$ ). Compared to the second treatment step in the STAR*D study, (remission rate $25 \%$ ), remission rates of the present study were to some extent higher. Possible explanations are the multiprofessional treatment approach including high additional effords such as psychotherapy, occupational therapy or physical therapy in the current study. However, comparing only the treatment step 2 of STAR*D (AD-Switch or -combination) with the corresponding treatment groups of the present study, remission rates do approach comparable numbers (remission rate [HRSD]: AD-comb: 42.9\%; AD-Switch: $40.7 \%)$.

Focussing on the differences between the 4 treatment groups in step 2, it is notable that patients who received a non-antidepressant as the second step (lithium or SGAs) had lower depressive symptoms than subjects who received continued treatment with antidepressants (switch or combination). The good outcome of the lithium augmented patients is in line with empirical evidence for this treatment approach [9].

Also augmentation with SGA showed lower scores of depressive symptoms compared to AD-Switch and AD-Comb. Response rates were relatively high compared to previous studies. Bauer et al. (2009) found response rates of 55\% for augmentation after nonresponse to antidepressant monotherapy [26]. For antidepressant augmentation with aripiprazole [27] and olanzapine [28], response rates were $33.7 \%$ and $25 \%$, respectively. In the metaanalysis of Nelson and Papakostas (2009) overall efficacy of augmentation with SGA in treatment-resistant depression has been proven and is in line with the data of our naturalistic study. There has been a lot of research regarding possible antidepressant effects of SGA focusing on the multiple effects of those substances on different neurotransmitters and their second messengers.

The mediocre outcome of switching the antidepressant in this naturalistic study is in line with recent findings of RCTs showing that there is no evidence supporting this strategy [5]. Instead there are data suggesting inferiority of continuing the hitherto not effective antidepressant [8]. Also AD-Comb was part of the second step of STAR*D: The combination included citalopram and bupropion with remission rates of $30 \%$. But RCTs only found superiority for reuptake-inhibitors in combination with alpha2-antagonists compared to placebo $[29,30]$.

In summary, although AD-Switch and AD-Comb show moderate remission rates in the present study, they might produce lower improvement and lower remission rates compared to Li-Augm or augmentation with SGAs. As noticed before, a possible reason for this is the relatively similar way of functioning of ADs, increasing the levels of serotonin and/or norepinephrine in the synaptic cleft (with few exceptions). However, antidepressant agents differ in the mode of action and specificity of increasing neurotransmitters. A possible conclusion could be that patients who do not respond to substances with this mode of functioning do have a higher benefit with medications of a different mode of action. Possible explanations of superiority of Li-Augm compared to $\mathrm{AD}$-Switch or $\mathrm{AD}$-Comb include the diversity of pharmacological functioning: Lithium influences the neurotransmittersystem on the level of second messengers and gene-regulation [31]. Previous research has also focused on possible antidepressant effects of SGAs particularly on the multiple effects of those substances on different neurotransmitters and their second messengers. For example, aripiprazole has a partial dopamine receptor agonism and quetiapine an additional norepiphreninreuptake-inhibition, which both are relevant in other antidepressant substances $[32,33]$. In contrast, $A D$ just have impact on extracellular receptors with a minor influence on multiple neurotransmitters.

\section{Conclusion}

$\nabla$

This naturalistic study confirmed that lithium augmentation as well as augmentation with SGA each showed better outcome than $\mathrm{AD}-\mathrm{Switch}$ and $\mathrm{AD}-\mathrm{Comb}$. Possible reasons are the diversity of functioning compared to the homologous effects in the group of antidepressants. However, there is nevertheless the risk of additional side effects of these 2 medication groups. Lithium treatment should be conducted in line with the established safety procedures. SGA and their effect on metabolic function as well as tardive dyskinesias are present and are criticized as underestimated [34]. Especially for the group of SGA the costs of medication should be taken into account. Therefore different treatment options, for example, additional psychotherapy or ECT $[35,36]$ should be considered. In conclusion, this study indicates differences between treatment strategies after failure of primary treatment in unipolar depression. RCTs are absolutely necessary to confirm these findings. 


\section{Limitations}

Despite the statistical control for influencing variables (medication, psychosocial factors, additional therapies, duration of treatment), the naturalistic design (no randomization to treatment groups) of the study can be regarded as a shortcoming and could influence the results. The lack of a defined measurement of non-response after phase I is also a major limitation. Therefore it is possible that not all patients really did show nonresponse to primary treatment. Furthermore the heterogeneous treatment in phase I could have had an influence on treatment outcome. Phase II was heterogeneous in treatment as well (different SGAs, different switch strategies, different AD combinations). Pharmacological differences of ADs were not fully considered in the question of the AD-comb or AD-switch. Therefore inferiority of those strategies could have been influenced by irrational switching or combination strategies. However, there is low evidence of efficacy of switching [5] or combination strategies [7] at all, and especially with regard to certain combinations or switching sequences. Another relevant aspect is that measurement at $\mathrm{t} 2$ was at discharge and not on a predefined time point. Although there were no significant differences of treatment duration between the 4 treatment arms, this could have had an influence on the results. In general it should be mentioned that there was no placebo group. Both groups with augmentation strategies received prolonged treatment with one antidepressant. It cannot be excluded that patients in these groups had lower depressive symptoms mainly because of prolonged exposure to the antidepressants. In conclusion, there is the possibility that treatment groups are not comparable. In further research there also should be an arm that continues the last treatment to observe effects of longer treatment and of spontaneous response. Therefore, the results in this naturalistic study indicate differences between these treatment strategies but all significant associations must be viewed with caution.

Nevertheless, the present study is the first study that compared different pharmacological treatment options after failure of primary antidepressant monotherapy with each other. Despite the methodological limitations mentioned, this study under naturalistic condition is an important completion to findings from randomised and controlled studies [37,38]. Either way, controlled studies, e.g., with randomization are required to confirm the findings from this study.

\section{Conflict of Interest}

\section{$\nabla$}

Yes: TB received lecture fees from Lilly, Bristol-Myers-Squibb, Lundbeck, esparma and AstraZeneca.

Affiliations

${ }^{1}$ Department of Psychiatry, Charité University Medicine Berlin, Campus Mitte, Berlin, Germany

${ }^{2}$ Department of Psychiatry and Psychotherapy, Kliniken im TheodorWenzel-Werk, Berlin, Germany

${ }^{3}$ Department of Psychiatry and Psychotherapy, Vivantes WenckebachKlinikum, Berlin, Germany

${ }^{4}$ Department of Psychology, Humboldt-University, Berlin, Germany ${ }^{5}$ Department of Psychiatry, Schlosspark-Klinik, Berlin, Germany and Department of Psychiatry, University Hospital, Technical University Dresden, Dresden, Germany

\section{References}

1 Rush AJ, Trivedi MH, Wisniewski SR et al. Acute and longer-term outcomes in depressed outpatients requiring one or several treatment steps: a STAR*D report. Am J Psychiatry 2006; 163: 1905-1917

2 Judd LL, Paulus MJ, Schettler PJ et al. Does incomplete recovery from first lifetime major depressive episode herald a chronic course of illness? Am J Psychiatry 2000; 157: 1501-1504

3 Bschor T. Therapy-resistant depression. Expert Rev Neurother 2010; 10: 77-86

4 Connolly KR, Thase ME. If at first you don't succeed: a review of the evidence for antidepressant augmentation, combination and switching strategies. Drugs 2011; 71: 43-64

5 Bschor T, Baethge C. No evidence for switching the antidepressant: systematic review and meta-analysis of RCTs of a common therapeutic strategy. Acta Psychiatr Scand 2010; 121: 174-179

6 Papakostas GI, Fava M, Thase ME. Treatment of SSRI-resistant depression: a meta-analysis comparing within- versus across-class switches. Biol Psychiatry 2008; 63: 699-704

7 Thase ME. Antidepressant combinations: widely used, but far from empirically validated. Can J Psychiatry 2011; 56: 317-323

8 Souery D, Serretti A, Calati $R$ et al. Citalopram versus desipramine in treatment resistant depression: effect of continuation or switching strategies: a randomized open study. World J Biol Psychiatry 2011; 12: 364-375

9 Crossley NA, Bauer M. Acceleration and augmentation of antidepressants with lithium for depressive disorders: two meta-analyses of randomized, placebo-controlled trials. J Clin Psychiatry 2007; 68: 935-940

10 Nelson JC, Papakostas GI. Atypical antipsychotic augmentation in major depressive disorder: a meta-analysis of placebo-controlled randomized trials. Am J Psychiatry 2009; 166: 980-991

11 Nierenberg $A A$. Switch or augment? Lessons from STAR*D. Ann Clin Psychiatry 2011; 22 (4 Suppl 3): S4-S8

12 Markowitz JC. Evidence-based psychotherapies for depression. J Occup Environ Med 2008; 50: 437-440

13 Willich S. Randomisierte kontrollierte Studien - pragmatische Ansätze erforderlich. Dtsch Ärzteblatt 2006; 103: A2524-A2529

14 Kaptchuk TJ. The double-blind, randomized, placebo-controlled trial: gold standard or golden calf? J Clin Epidemiol 2001; 54: 541-549

15 Hamilton M. A rating scale for depression. J Neurol Neurosurg Psychiatry $1960 ; 23: 56-62$

16 Hiller W, Zaudig M, Mombour W. IDCL: ICD-10 Checklists. Göttingen: Hogrefe; 2005

17 DGPPN B, KBV, AWMF, AkdÄ, BPtK, BApK, DAGSHG, DEGAM, DGPM, DGPS, DGRW for the guideline group unipolar depression*. S3-Guideline/National Guideline for unipolar depression (in German). Berlin, Düsseldorf: DGPPN, ÄZQ AWMF; 2009 [cited 2009]; 1. [available from: www.dgppn.de, www.versorgungsleitlinien.de, www.awmfleitlinien.de ]

18 Beck AT, Ward $\mathrm{CH}$, Mendelson $M$ et al. An inventory for measuring depression. Arch Gen Psychiatry 1961; 4: 561-571

19 Derogatis $L R$, Melisaratos $N$. The brief symptom inventory: an introductory report. Psychol Med 1983; 13: 595-605

20 Endicott J, Spitzer RL, Fleiss JL et al. The global assessment scale. A procedure for measuring overall severity of psychiatric disturbance. Arch Gen Psychiatry 1976; 33: 766-771

21 Guy W. Clinical Global Impression. In: Guy W (ed.). ECDEU Assessment Manual for Psychopharmacology. 1976

22 First M, Gibbon M, Spitzer R. Structured Clinical Interview for DSM-IV Axis II Disorders. New York NY: Biometrics Research Dept, NewYork State Psychiatric Institute; 1996

23 Olejnik S, Algina J. Measures of effect size for comparative studies: applications, interpretations, and limitations. Contemp Edu Psychol 2000; 25: 241-286

24 CIPS. International scales for psychiatry. (CIPS) CIPS (ed.). Göttingen: Beltz Test GmbH; 2005

25 Seemüller F, Riedel M, Obermeier $M$ et al. Outcomes of 1014 naturalistically treated inpatients with major depressive episode. Eur Neuropsychopharmacol 2010; May; 20 (5): 346-355 Epub 2010 Jan 22

26 Bauer M, Pretorius HW, Constant EL et al. Extended-release quetiapine as adjunct to an antidepressant in patients with major depressive disorder: results of a randomized, placebo-controlled, double-blind study. J Clin Psychiatry 2009; 70: 540-549

27 Berman RM, Marcus RN, Swanink $R$ et al. The efficacy and safety of aripiprazole as adjunctive therapy in major depressive disorder: a multicenter, randomized, double-blind, placebo-controlled study. J Clin Psychiatry 2007; 68: 843-853 
28 Trivedi $M H$, Thase ME, Osuntokun $O$ et al. An integrated analysis of olanzapine/fluoxetine combination in clinical trials of treatmentresistant depression. J Clin Psychiatry 2009; 70: 387-396

29 Carpenter LL, Yasmin S, Price LH. A double-blind, placebo-controlled study of antidepressant augmentation with mirtazapine. Biol Psychiatry 2002; 51 : 183-188

30 Blier P, Ward HE, Tremblay $P$ et al. Combination of antidepressant medications from treatment initiation for major depressive disorder: a double-blind randomized study. Am J Psychiatry 2010; 167: 281-288

31 Wada A. Lithium and neuropsychiatric therapeutics: neuroplasticity via glycogen synthase kinase-3beta, beta-catenin, and neurotrophin cascades. J Pharmacol Sci 2009; 110: 14-28

32 Davies MA, Sheffler DJ, Roth BL. Aripiprazole: a novel atypical antipsychotic drug with a uniquely robust pharmacology. CNS Drug Rev 2004; 10: 317-336

33 Pira $L$, Mongeau $R$, Pani $L$. The atypical antipsychotic quetiapine increases both noradrenaline and dopamine release in the rat prefrontal cortex. Eur J Pharmacol 2004; 504: 61-64
34 Carroll BJ. Antipsychotic drugs for depression? Am J Psychiatry 2010; 167: 216 author reply 219

35 McCullough JP Jr. Treatment for chronic depression using cognitive behavioral analysis system of psychotherapy (CBASP). J Clin Psychol 2003; 59: 833-846

36 UK ECT Review Group. Efficacy and safety of electroconvulsive therapy in depressive disorders: a systematic review and meta-analysis. Lancet 2003; 361: 799-808

37 Mulder RT, Frampton C, Joyce PR et al. Randomized controlled trials in psychiatry. Part II: their relationship to clinical practice. Aust N Z J Psychiatry 2003; 37: 265-269

38 Porter R, Frampton C, Joyce PR et al. Randomized controlled trials in psychiatry. Part 1: methodology and critical evaluation. Aust N Z J Psychiatry 2003; 37: 257-264 